\title{
PROPUESTA DIDÁCTICA CON ENFOQUE ECOLÓGICO E INTERDISCIPLINAR: UNA APUESTA POR LA EDUCACIÓN SUPERIOR INCLUSIVA ${ }^{1}$
}

\section{Didactic Proposal with Ecological and Interdisciplinary Approach: a Commitment to Inclusive Higher Education}

Doris Adriana RAmírez SAlazAR

Universidad de Antioquia. Colombia

pandrea.soto@udea.edu.co

Paola Andrea Soto Ossa

Universidad de Antioquia. Colombia

María Teresa Rugeles López

Universidad de Antioquia. Colombia

Luz Helena Lugo Agudelo

Universidad de Antioquia. Colombia

Carlos Alberto QuinTero Valencia

Universidad de Antioquia y Comité de Rehabilitación de Antioquia. Colombia

María Catalina Ocampo Agudelo

Universidad de Antioquia. Colombia

Marcela Ruiz YEPES

Universidad Católica Luis Amigó. Colombia

Diego Andrés Pereira Velásquez

Comité de Rehabilitación de Antioquia. Colombia

1 Este artículo es derivado de un proyecto de investigación financiado por Colciencias y la Universidad de Antioquia entre 2016 y 2018, y desarrollado por los grupos de investigación Didáctica y Nuevas Tecnologías y Rehabilitación en Salud, en asocio con el Comité de Rehabilitación. 
Alejandra VANegas Castro

Universidad Pontificia Bolivariana. Colombia

Carolina Lopera Cataño

Universidad de Antioquia. Colombia

Vanessa Andreina Seijas Bermúdez

Universidad de Antioquia. Colombia

Recepción: 19 de marzo de 2019

Aceptación: 12 de noviembre de 2020

Resumen: Las personas en situación de discapacidad intelectual han tenido pocas oportunidades para acceder a la educación superior; durante mucho tiempo se ha considerado que ellas no son un colectivo potencial para formarse en el ámbito universitario. Este artículo es derivado de una investigación que se desarrolló en el contexto colombiano, cuyo propósito consistió en contribuir a disminuir las barreras de los jóvenes para su inclusión en el contexto familiar, social, académico y ocupacional, mediante el diseño y validación de un modelo de atención integral con enfoque ecológico e interdisciplinar. El estudio tuvo una perspectiva metodológica mixta, se utilizó la escala ABAS II para evaluar el índice de conducta adaptativa en 20 jóvenes a modo de pretest y postest. Se concluyó que esta experiencia contribuyó a la formación y calidad de vida de los jóvenes; y mostró que a todas las personas se les puede enseñar, pero que cada una aprende en su singularidad.

Palabras Clave: jóvenes en situación de discapacidad intelectual; transición a la vida adulta; inclusión; modelo de atención integral con enfoque ecológico e interdisciplinar; educación superior inclusiva.

Aвstract: People with intellectual disabilities have had few opportunities to access higher education, for a long time it has been considered that they are not a potential group to be trained in the university environment. This article is derived from an investigation that was developed in the Colombian context, the purpose of which was to contribute to reducing the barriers of young people for their inclusion in the family, social, academic and occupational context, through the design and validation of a model of comprehensive care with an ecological and interdisciplinary approach. The study had a mixed methodological perspective, the ABAS II scale was used to evaluate the adaptive behavior index in 20 young people as a pretest and posttest. It was concluded that this experience contributed to the formation and quality of life of the young people; and it showed that all people can be taught, but that each one learns in their uniqueness.

KEYWORDS: young people with intellectual disabilities; transition to adult life; inclusion; comprehensive care program with an ecological and interdisciplinary approach; inclusive higher education model. 
PROPUESTA DIDÁCTICA CON ENFOQUE ECOLÓGICO E INTERDISCIPLINAR:

UNA APUESTA POR LA EDUCACIÓN SUPERIOR INCLUSIVA

D. A. RAMÍrEZ, P. A. SOTO, M. $^{a}$ T. RUGElES, L. H. LUGO, C. A. QUINTERO, M. ${ }^{a}$ C. OCAMPO ET AL.

\section{Introducción y estado de la cuestión}

L

A EDUCACIÓN ES UN DERECHO, incluso, para las personas con discapacidad, que no se limita únicamente a la formación preescolar, básica primaria, básica secundaria y media (Chaves, 2018). No obstante, las personas en situación de discapacidad intelectual han tenido pocas oportunidades para acceder a la educación superior, durante mucho tiempo se ha considerado que ellas no son un colectivo potencial para formarse en el ámbito universitario.

En la Convención Internacional sobre los Derechos de las Personas con Discapacidad, ratificada en Colombia en el año 2009 a través de la Ley 1346, se hace énfasis en que los Estados deben garantizar el derecho a la educación desde el nivel inicial hasta el superior, y deben hacerlo desde los principios de accesibilidad para todos, sin discriminación de raza, género, edad, lengua, religión, ni por diferencias de orden social, económico, político, religioso o por discapacidades (Cobos y Moreno, 2014). Este marco normativo busca disminuir la desventaja social de la persona en situación de discapacidad y promover su participación en igualdad de oportunidades en diferentes escenarios teniendo en cuenta sus derechos (Díez y Sánchez, 2015). El acceso a la educación es un derecho fundamental que posibilita el desarrollo personal y social de los individuos, y la Universidad ha de constituirse en un escenario abierto a la diversidad de las personas que en ella habitan (Bernal, 2014).

A pesar de la existencia de un marco normativo que promueve la educación igualitaria para todas las personas, existe una brecha entre las políticas y las acciones inclusivas que desarrollan las Instituciones de Educación Superior (IES) en el contexto colombiano. Se han observado avances importantes con respecto a las políticas inclusivas que se han diseñado para orientar y regular la atención educativa que se le ofrece a la población con discapacidad. Por ejemplo, a partir del Decreto 1421 se declaró que todos los estudiantes con alguna condición de discapacidad deben acceder a la oferta institucional existente, con estudiantes de su edad y recibir los apoyos y ajustes razonables pertinentes para que su proceso educativo sea exitoso y mejore su calidad de vida (MEN, 2017). Sin embargo, esta directriz no se corresponde con la situación en la que se encuentran todos los estudiantes que ingresan a la universidad (Chaves, 2018).

Esta situación evidencia que Colombia está aún en una fase de cambios iniciales, y es necesario continuar trabajando para garantizar el cumplimiento del derecho a la educación de las personas en situación de discapacidad. De acuerdo con Ramírez (2018), existe un lento reconocimiento de la educación como un derecho de todas las personas; ante esta situación, se han creado diferentes políticas públicas e instrumentos legales que buscan proteger a las personas con discapacidad y ofrecerles garantías para que puedan acceder a la educación.

Aunque se evidencia un mayor compromiso por parte de las IES para promover procesos inclusivos, se observa que las prácticas de educación superior con las personas que presentan alguna discapacidad son aún incipientes. Si bien "se han venido desarrollando estrategias administrativas, curriculares, docentes e investigativas, se evidencia la desarticulación entre dichos mecanismos, ocasionando que la población 
con discapacidad en la institución no sienta directamente el impacto de estas acciones” (Ramírez, 2018, p. 51).

Se hace necesario entonces que las IES garanticen el acceso a la educación de las personas en situación de discapacidad, pero es fundamental que se rompa el esquema "en el cual estos estudiantes deben adaptarse al sistema, y más bien, adaptar la universidad a los estudiantes, superando los obstáculos que les dificultan su acceso, permanencia y egreso satisfactorios” (Chaves, 2018, p. 85). Es evidente, entonces, la necesidad de implementar acciones que consoliden políticas y prácticas inclusivas y derrumben las barreras para la inclusión familiar, social, educativa y ocupacional de los jóvenes en situación de DI. Es pertinente avanzar en el proceso de transformación de políticas, culturas y prácticas para comprender y abordar la discapacidad desde una óptica que posibilite la generación de líneas de acción, que promuevan la participación, la inclusión y el desarrollo integral de los jóvenes en la sociedad.

Por ello, la Universidad de Antioquia, a través de los grupos de investigación Didáctica y Nuevas Tecnologías de la Facultad de Educación y Rehabilitación en Salud de la Facultad de Medicina, se vincularon con el Comité de Rehabilitación y propusieron un programa de formación denominado UIncluye, el cual se desarrolló en tres fases, y buscó contribuir a disminuir las barreras de los jóvenes en situación de DI para su inclusión en el contexto familiar, educativo, social y ocupacional, mediante el diseño y validación de un modelo de atención integral con enfoque ecológico e interdisciplinar.

La primera fase, denominada Escalar, se estructuró con el propósito de preparar a las familias y a los jóvenes para el ingreso a la vida adulta y favorecer su vinculación al entorno universitario. Durante seis meses se abordó un currículo centrado en el desarrollo de habilidades para la vida en comunidad y la participación ciudadana; la comunicación y la interacción; el desplazamiento y la movilidad, utilizando el transporte público; la gestión financiera, y la alfabetización funcional. La fase Escalar se centró en el desarrollo de actividades académicas y extracurriculares necesarias para la vida adulta, teniendo en cuenta las necesidades, habilidades, intereses y apoyos que requería cada joven (Pallisera, 2011). En este sentido, se buscó que los jóvenes lograran objetivos relacionados con la participación en diversas actividades formativas, más allá de la educación media obligatoria; con el aprendizaje de habilidades de autodeterminación, la implicación y participación activa en diferentes escenarios de la comunidad y la posibilidad de experimentar relaciones sociales y personales satisfactorias (Pallisera et al., 2014).

Durante la segunda fase, denominada UIncluye, se consolidó e implementó un currículo que incluyó acciones y cursos organizados en tres núcleos: social-práctico, conceptual y vocacional-ocupacional. Los jóvenes participaron en actividades en estos tres núcleos que se conjugaron con acciones transversales de un taller artístico y acciones específicas en tres ejes temáticos: ciencias alimentarias, ciencias veterinarias y agrarias y pedagogía. Este itinerario formativo les permitió a los jóvenes fortalecer su conducta adaptativa, habilidades conceptuales, sociales y prácticas; los involucró en actividades que les posibilitaron acercarse de manera directa a la universidad, interactuar en espacios de ciudad y reconocerse como seres humanos que transitan de la 
adolescencia a la vida adulta, etapa que les demandó nuevos retos y les exigió revisar y ajustar sus roles personales, familiares y sociales. Esta etapa los preparó para asumir con responsabilidad y mayor autonomía su vida adulta consigo mismos, en la familia, en la universidad y en la sociedad en general.

Durante la tercera fase, denominada Diplomas, se buscó promover en cada uno de los jóvenes habilidades sociales, personales y académicas para realizar de forma más autónoma las acciones relacionadas con una ocupación, y que facilitaran su inclusión en los diferentes contextos familiares, universitarios, comunitarios y sociales. El itinerario formativo desarrolló las habilidades de comunicación, autodirección, habilidades académicas funcionales, habilidades sociales, de ocio y tiempo libre, autocuidado, utilización de los recursos comunitarios, y salud y seguridad. En esta fase se fortaleció el proceso de articulación con las facultades de medicina veterinaria y ciencias agrarias y química farmacéutica y ciencias alimentarias para el diseño y ejecución de los talleres vocacionales-ocupacionales.

Esta propuesta de formación se centró en reconocer que a todas las personas se les puede enseñar, pero que cada una aprende en su singularidad. Esto implica comprender la diferencia desde una perspectiva relacional; sin concentrarse en el déficit, la falta y la dificultad del individuo, sino en el medio. Se requiere entonces "una transformación ética que se desplace de la mirada sobre aquellos sujetos apuntados como diferentes y pase a ser una mirada puesta en un nosotros, en todo aquello que pasa -pedagógicamente- entre nosotros" (Skliar, 2015, p. 43).

\section{Referentes teórico-conceptuales que sustentan el programa de formación}

La DI se define como un estado individual que se caracteriza por presentar limitaciones significativas tanto en el funcionamiento intelectual como en la conducta adaptativa (CA), y es originado antes de los 18 años (Flórez, 2018). No se comprende como un rasgo absoluto o fijo de la persona, sino que se fundamenta en considerar principalmente la interacción de la persona con su entorno, y el efecto que los apoyos pueden proporcionar para fortalecer su desempeño en el ámbito social (Cuesta et al., 2019). Esta concepción en torno a la DI pone énfasis en las capacidades y potencialidades de las personas y en los apoyos que necesitan para optimizar su funcionamiento individual y tener mayores posibilidades de participación en todos los ámbitos de la vida social (Cuesta et al., 2019; Vicente-Sánchez et al., 2018).

Existen algunas barreras que limitan e impiden la participación de los jóvenes en las IES. Los modelos de inclusión en el campo de la educación se han ido adoptando paulatinamente, principalmente en los niveles de educación básica y media, pero esto no ha sucedido en igual medida en la educación superior (Ramírez, 2018). Así mismo, se ha considerado que a las universidades solo pueden acceder personas con altas capacidades intelectuales, desconociendo las habilidades que puede desarrollar y fortalecer un joven en situación de DI en un ambiente universitario (Zacarías et al., 2015). Durante años han prevalecido algunas creencias derivadas del modelo médico rehabilitatorio que considera a la discapacidad como una enfermedad, lo que deriva 
PROPUESTA DIDÁCTICA CON ENFOQUE ECOLÓGICO E INTERDISCIPLINAR:

UNA APUESTA POR LA EDUCACIÓN SUPERIOR INCLUSIVA

D. A. RAMÍrEZ, P. A. SOTO, M. $^{a}$ T. RUGElES, L. H. LUGO, C. A. QUINTERO, M. ${ }^{a}$ C. OCAMPO ET AL.

en que no se le proporcionen los apoyos adecuados a todas las personas para que puedan avanzar hacia una mayor calidad de vida (Zacarías et al., 2015) y participar en diferentes escenarios (Cuesta et al., 2019).

Por su parte, en los niveles de la educación básica y media no se promueve el desarrollo de las habilidades académicas y sociales en los estudiantes, ya que no se precisan ajustes razonables como la utilización de metodologías diversas, el uso de recursos que faciliten el acceso a los contenidos, ni tampoco se adaptan las pruebas de evaluación (Moriña y Cotán, 2017). Además, algunos estudiantes tienen dificultades para establecer relaciones sociales, interactuar con los demás y ser incluidos en los diferentes escenarios escolares (Delgado, 2015); razones por las cuales muchos de ellos terminan desertando de su proceso escolar (Claro, 2007). De igual manera, algunos estudiantes han sido promovidos de grado de forma automática, sin tener en cuenta el estado real de sus habilidades académicas (Kremer y Quijano, 2015).

En los casos en los que los jóvenes logran ingresar a la Universidad, se evidencia la ausencia de directrices que marquen un protocolo que garantice el éxito en este cambio de etapas, lo que provoca que el estudiante "viva esta etapa con inestabilidad e incertidumbre, generando con ello bajo rendimiento académico, motivos de abandono escolar o toma de decisiones contrarias a su orientación vocacional o profesional" (Alcaín y Medina, 2017, p. 12). Otra barrera para la inclusión en los estudios superiores de los jóvenes en situación de discapacidad es el desconocimiento y la escasa formación del profesorado en materia de discapacidad. Por ello, es fundamental que los profesores estén informados, sensibilizados y formados para atender adecuadamente a las necesidades de los estudiantes. Se requieren maestros que desarrollen prácticas educativas que respeten las diferencias de la comunidad académica, con o sin discapacidad (Barradas et al., 2019). Docentes bien formados, sin prejuicios y actualizados, que acojan a sus estudiantes y le den valor a la diversidad (Chaves, 2018).

En este contexto, es oportuno reconocer que el concepto de inclusión debe ser entendido como un medio para asegurar que todos los estudiantes con o sin discapacidad disfruten de los mismos derechos. "Es un proceso que demanda genuina participación y transformación de la comunidad universitaria e implica que las minorías no sean discriminadas en razón de sus diferencias” (Bernal, 2014, p. 1).

En la investigación se le concedió un lugar especial a la inclusión educativa de las personas en situación de discapacidad, por considerarse un derecho y una oportunidad para su desarrollo, ya que toda acción de inclusión educativa debería tener como fin primordial el logro de aprendizajes significativos en los estudiantes, lo cual supone no solo la adquisición de contenidos, sino la capacidad para resolver problemas y aprender de manera permanente. Para ello, es necesario avanzar en el "proceso de reestructuración de la dinámica y el enfoque de trabajo de las instituciones, tanto en los procesos organizacionales, de servicio y apoyo, como en los académicos, curriculares, pedagógicos y de aprendizaje" (Bernal, 2014, p. 56).

La Educación Superior Inclusiva es un movimiento que se fundamenta en el principio de educación para todos, y se reconoce como un derecho inalienable; que busca garantizar una educación de calidad y ofrecer diferentes espacios de participación para todos los estudiantes (Cotán, 2017; Ramírez, 2018). Este enfoque promueve que

Ediciones Universidad de Salamanca / CC BY-NC-ND

Siglo Cero, vol. 52 (3), 2021, julio-septiembre, pp. 163-184 
PROPUESTA DIDÁCTICA CON ENFOQUE ECOLÓGICO E INTERDISCIPLINAR:

UNA APUESTA POR LA EDUCACIÓN SUPERIOR INCLUSIVA

D. A. RAMÍrEZ, P. A. SOTO, M. $^{a}$ T. RUGElES, L. H. LUGO, C. A. QUINTERO, M. ${ }^{a}$ C. OCAMPO ET AL.

las instituciones acepten las diferencias de su comunidad y se adapten a ellas, para que todas las personas puedan acceder y participar en los distintos escenarios sociales, laborales y de formación profesional (Chaves, 2018; Moriña y Carballo, 2018; Ocampo, 2011).

El paradigma de los apoyos tiene un impacto en el funcionamiento individual de las personas en situación de DI. Cada joven es diferente, y demuestra en su funcionamiento cotidiano tanto habilidades como dificultades distintas en intensidad y en cualidad, en relación con otras personas (Muntaner, 2010). Por ello, es fundamental identificar sus puntos fuertes y débiles, para valorar su potencial y sus posibilidades de desarrollo, lo que implica que se ofrezcan servicios que les ayuden a desarrollar sus capacidades, y, a su vez, que den respuesta a sus necesidades (Verdugo, 1995). En este contexto, se reconoce que las personas en situación de DI pueden desarrollar sus potencialidades, más allá de las creencias o prejuicios que puedan tenerse (Chaves, 2018), lo más importante es la persona y no las condiciones de capacidad o limitación que tenga, ya que, con los apoyos pertinentes, el funcionamiento mejora, salvo en casos excepcionales (Flórez, 2018). Esto indica que toda persona puede progresar permanentemente si se le ofrecen los apoyos adecuados y de forma sostenida; es un reto del entorno proporcionar diferentes sistemas de apoyo que hagan que mejore su calidad de vida (Tamarit, 2010).

Los apoyos se definen como recursos y estrategias que buscan promover el desarrollo, la educación, los intereses y el bienestar de un individuo (Tamarit, 2010). La provisión de apoyos permite que la persona pueda realizar actividades cotidianas y disfrutar del ejercicio de sus derechos, pero esto no implica que, pasado un tiempo, la persona no vaya a necesitar más apoyos, en tanto estos le permiten desenvolverse mejor, pero no son absolutamente eliminables (Cuesta et al., 2019).

Por su parte, el proceso de transición a la vida adulta se constituye en una etapa fundamental en la vida de las personas. Para Pallisera et al. (2014), el proceso de transición a la vida adulta va más allá del tránsito de la escuela al trabajo; constituye un proceso complejo que incluye que los jóvenes participen en actividades formativas más allá de la educación secundaria; en actividades relacionadas con el cuidado y la organización de su hogar; se involucren activamente en distintos espacios de la comunidad; experimenten relaciones sociales y personales satisfactorias; trabajen, y, en definitiva, se favorezca el desarrollo de su autonomía tanto en el contexto familiar como en el social.

Son tres los elementos fundamentales que han de configurar cualquier proceso de transición a la vida adulta: un programa educativo que favorezca el desarrollo de aprendizajes significativos y útiles para la vida; un plan individualizado que contenga los componentes principales que llevan a la vida adulta; y opciones de empleo, vivienda y participación en la comunidad que les posibiliten a los jóvenes tomar decisiones y hacer elecciones (Jordán de Urriés y Verdugo, 2013). En definitiva, la transición a la Universidad y a la vida adulta es un proceso complejo que conlleva para los estudiantes múltiples y significativos cambios personales, los cuales se presentan porque existe una discontinuidad entre los niveles de formación secundaria y universitaria (Alcaín y Medina, 2017).

Ediciones Universidad de Salamanca / CC BY-NC-ND

Siglo Cero, vol. 52 (3), 2021, julio-septiembre, pp. 163-184 
En el marco de estos cambios que experimentan los jóvenes al transitar de una etapa formativa a otra, es oportuno promover en ellos habilidades de autodeterminación y planificar los apoyos teniendo en cuenta sus singularidades. La autodeterminación tiene gran relevancia en la vida de niños, jóvenes y adultos con y sin discapacidad (Vicente-Sánchez et al., 2018), y se refiere a que el individuo actúe como el principal agente causal de su vida, tome decisiones sin influencias externas, y es un proceso que se aprende en la interacción entre las capacidades que tiene una persona de escoger y actuar y las oportunidades que el contexto le ofrece para elegir y decidir (Muntaner, 2010).

La planificación centrada en la persona es una metodología basada en la colaboración, para ayudar a las personas en situación de DI a acceder a los apoyos y servicios para tener una mejor calidad de vida. Los jóvenes son el foco de la planificación de los apoyos, se tienen en cuenta las decisiones que tomen y se reconocen sus capacidades, expectativas, sueños, preferencias y valores (Arellano y Peralta, 2016; Carratalá et al., 2017). Es un proceso de escucha y aprendizaje continuo, centrado en lo que se espera del presente y no del futuro (Cuervo et al., 2017).

Finalmente, la CA está compuesta por tres dominios: conceptual, social y práctico. El conceptual se encuentra asociado a la comunicación, habilidades académicas funcionales y autodirección. Las habilidades de comunicación incluyen la capacidad para comprender y transmitir información, ideas, necesidades y deseos a través del lenguaje receptivo, expresivo y la comunicación no verbal. Las habilidades académicas funcionales comprenden la capacidad para leer, escribir y realizar operaciones matemáticas, las cuales son necesarias para un funcionamiento adaptativo en la sociedad (Miller y Fenty, 2013). Las habilidades de autodirección son necesarias para ejercer la independencia, la responsabilidad y el autocontrol personal; incluyen el manejo de horarios, la toma de decisiones y la realización de tareas de principio a fin (Harrison y Oakland, 2013).

El dominio social hace alusión a las habilidades sociales, las cuales se definen como conductas socialmente significativas que se manifiestan en determinadas situaciones (Park et al., 2013). Las habilidades de uso del tiempo de ocio hacen referencia al desarrollo de intereses variados que reflejan las preferencias y elecciones personales (Verdugo, 1995).

El dominio práctico tiene que ver con las habilidades de autocuidado, vida en el hogar y en la escuela, utilización de los recursos comunitarios, salud y seguridad, y empleo. Las habilidades de autocuidado son esenciales para el funcionamiento diario básico, participar en la comunidad y gozar de experiencias que eleven y mantengan la calidad de vida (Wallace y Shubert, 2013). Las habilidades de la vida en el hogar y en la escuela tienen que ver con el cuidado de las pertenencias, el mantenimiento del medio ambiente y la realización de actividades rutinarias en el hogar (Steere et al., 2013). Las habilidades para la utilización de los recursos comunitarios son fundamentales para que el individuo logre desenvolverse con facilidad en diferentes contextos, incluye las habilidades para hacer compras y movilizarse y la asistencia a diferentes espacios y eventos culturales (Dymond, 2013). La salud y la seguridad se refieren a las habilidades para la protección de la salud y para enfrentar enfermedades y lesiones; incluyen- 
do el cumplimiento de normas de seguridad y el uso adecuado de los medicamentos (Agran et al., 2013). Y las actitudes relacionadas con el empleo hacen alusión a poseer un trabajo en la comunidad; e incluye habilidades como finalizar tareas, conocer los horarios, seguir un plan de trabajo, manejar el dinero, interactuar con los compañeros y desplazarse a su lugar de trabajo de manera independiente (Mutua, 2013; Verdugo, 1995).

\section{Diseño metodológico}

\subsection{Sobre el método}

La investigación tuvo un diseño metodológico cuasiexperimental que incluyó un grupo experimental y la aplicación de pruebas antes y después de la intervención, de modo que durante el proceso de recolección y análisis se pudo obtener un control deliberado de las variables de interés. De acuerdo con Hernández et al. (2014), en estos casos: "Existe un punto de referencia inicial para ver qué nivel tenía el grupo en las variables dependientes antes del estímulo; es decir, hay un seguimiento del grupo" (p. 141).

Este estudio tuvo una perspectiva metodológica mixta, debido a que consideró tanto en el proceso de recolección de información como en el de análisis datos de orden cualitativo y cuantitativo. Se trató de un enfoque integrador de naturaleza complementaria y explicativa (Creswell, 2013). La implementación de los métodos cuantitativo y cualitativo en esta investigación respondió a un diseño añadido o incrustado concurrente, la integración de métodos se presentó en la fase de análisis. Este diseño recogió simultáneamente datos cuantitativos y cualitativos, pero existió un método predominante que orientó el proyecto; en este caso, el cuantitativo. Sin embargo, los datos de orden cualitativo actuaron como complemento para ampliar y dar mayor validez al estudio. Este diseño suele proporcionar una visión más amplia del fenómeno estudiado (Hernández et al., 2014).

\subsection{Sobre los participantes, los instrumentos y el procedimiento}

Los participantes se seleccionaron teniendo en cuenta los siguientes criterios: diagnóstico de DI, un coeficiente intelectual (CI) por debajo de 70 o 75 puntos; informe de la CA del joven elaborado por el colegio o la institución que lo remitía; tener entre 18 y 26 años; haber culminado el grado $11 .^{\circ}$, o al menos el grado noveno de educación regular, y tener un nivel funcional de lectura, escritura y pensamiento lógico matemático.

El grupo estuvo conformado por 20 jóvenes; el $55 \%$ eran mujeres y el $45 \%$ hombres, cuyas edades oscilaban entre 18 y 26 años. Los jóvenes debían presentar limitaciones significativas en su funcionamiento intelectual y en la CA; para ello, algunos presentaron la prueba de CI y otros los informes de la CA. En cuanto a la escolaridad, 
el $65 \%$ de los jóvenes eran bachilleres; el $15 \%$ se encontraban cursando los grados 10 y 11 , y el $20 \%$ no habían finalizado el proceso de educación básica secundaria; y a pesar de ello se incluyeron en el grupo base porque, al igual que los demás jóvenes, demostraron tener un nivel funcional de lectura, escritura y pensamiento lógico matemático. Los jóvenes tenían un nivel funcional de lectura, escritura y pensamiento lógico matemático, pero cada uno de ellos requería que se le acompañara de manera particular para el desarrollo de las actividades. Por ello, se optó por organizarlos según los niveles de apoyo que requerían.

El $45 \%$ de los jóvenes requerían apoyos intermitentes o limitados cuando tenían preguntas específicas sobre la tarea que se encontraban desarrollando, y generalmente terminaban sus actividades a partir de las orientaciones generales que se les brindaban. El $30 \%$ de los jóvenes requerían apoyos extensos para iniciar las actividades, y apoyos intermitentes para continuarlas y finalizarlas. Los demás, el $25 \%$, requerían apoyos extensos y generalizados para iniciar, continuar y finalizar las actividades que se les proponían en los diferentes escenarios de formación.

También participaron en la investigación otras 79 personas: nueve profesores de la Facultad de Ciencias Farmacéuticas y Alimentarias; diez profesores de la Facultad de Ciencias Agrarias; una profesora que acompañó los talleres de huertas; nueve profesores que ofrecieron talleres de actividad física, sexualidad, expresión vocal, expresión corporal, prevención de riesgos, entre otros; cinco profesionales de apoyo (coordinadora académica, licenciada en educación especial, psicóloga, trabajadora social y terapeuta ocupacional); ocho estudiantes en formación (2 estudiantes de posgrado y 6 estudiantes de la licenciatura en Educación Especial), y treinta y siete familiares de los estudiantes ( 20 madres de familia, 9 padres de familia, 2 padrastros, 3 hermanos, 1 prima, 1 abuela y 1 tía).

En cuanto a las técnicas e instrumentos que se utilizaron para generar la información, se utilizó la escala ABAS II para evaluar el índice de CA en los jóvenes. Los datos se recolectaron durante el periodo comprendido entre el 2017 y el 2018. El instrumento se aplicó en dos momentos, el primer momento fue previo a la fase Escalar y la segunda aplicación al finalizar el programa de formación UIncluye, es decir, 16 meses después. Se analizó la información teniendo como punto de referencia las medianas (diferencias entre pt1-pt0), lo que permitió identificar un avance significativo en los jóvenes en relación con las habilidades funcionales cotidianas necesarias para desenvolverse de manera autónoma en los diferentes escenarios.

Las diferencias en el índice de CA arrojado por el instrumento ABAS-II antes y después de iniciar la propuesta de formación se analizaron con la prueba estadística de Wilcoxon. La correlación entre las valoraciones que se obtuvieron del autoinforme y del heteroinforme se analizaron usando el coeficiente de correlación de Spearman. Todas las pruebas estadísticas fueron de una cola y un valor $\mathrm{p}<0,05$, que fue considerado como estadísticamente significativo.

Este instrumento fue aplicado por la licenciada en Educación Especial, la psicóloga y las estudiantes en formación, quienes fueron previamente formadas por el equipo investigador. La escala fue diligenciada por las personas cercanas a los jóvenes que se encontraban en disposición de valorar sus habilidades adaptativas (heteroinforme) y 
por los mismos jóvenes (autoinforme). Todos los participantes fueron informados sobre su participación en el estudio y, con anterioridad a la aplicación de la escala, se les compartió el consentimiento informado en el cual se les comunicaban los objetivos del estudio y se les explicaba que su participación en el estudio era voluntaria y que la información recopilada se utilizaría únicamente con fines académicos.

Para el análisis del perfil cuantitativo que arrojó el instrumento se retomaron los dos tipos de puntuaciones normativas: las puntuaciones escalares y las típicas, las cuales permitieron realizar la interpretación y comparar los resultados. Las puntuaciones escalares de las áreas de habilidad se distribuyen con media de 10 y desviación típica de 3. Las puntuaciones típicas se calculan de acuerdo con los dominios de la CA y al índice de CAG, y se distribuyen con media de 100 y desviación típica de 15.

\begin{tabular}{|l|c|c|c|c|c|c|c|}
\hline \multicolumn{7}{|c|}{ Tabla 1. Puntuaciones escalares y típicas } \\
\hline $\begin{array}{l}\text { CAG y dominios } \\
\text { (Categoría descriptiva } \\
\text { en niveles de } \\
\text { rendimiento) }\end{array}$ & Muy baja & Baja & Media-baja & Media & Media-alta & Alta & Muy alta \\
\hline Puntuación & $50-70$ & $71-79$ & $80-89$ & $90-109$ & $110-119$ & $120-129$ & $130-150$ \\
\hline $\begin{array}{l}\text { Áreas de habilidad } \\
\text { (Categoría descriptiva } \\
\text { en niveles de } \\
\text { endimiento) }\end{array}$ & Muy baja & Baja & Media-baja & Media & Media-alta & Alta \\
\hline Puntuación & 3 o menos & 4 a 5 & 6 a 7 & 8 a 12 & 13 a 14 & 15 y más \\
\hline
\end{tabular}

Adicionalmente, se utilizó una matriz de observación para comprender lo que se ve, y aun aquello que no puede verse a simple vista (Simons, 2011). Se hizo uso del diario de campo y la matriz de seguimiento para registrar el proceso de los participantes en el Programa UIncluye, todos los instrumentos fueron diligenciados por las licenciadas en Educación Especial, la psicóloga y el terapeuta ocupacional.

\section{Descripción del programa de formación}

El programa se desarrolló en tres fases que se llevaron a cabo durante 16 meses, su propósito consistió en contribuir a que los jóvenes pudieran acceder al entorno universitario, participar en el ámbito social, tener una vida autónoma y disfrutar de una mayor calidad de vida (Vived et al., 2012).

Desde el comienzo de este proyecto se reconoció que todo proceso de transición a la vida adulta responde a una planificación detallada, en tanto solo a través de una programación coordinada y continua será posible que los jóvenes en situación de DI puedan integrarse al entorno universitario y a la vida en sociedad (Jiménez y Verdugo, 1999). La propuesta curricular se centró en favorecer el desarrollo de habilidades en los jóvenes que les permitieran vivir en comunidad y ser más autónomos e indepen- 
dientes. Se fortalecieron habilidades conceptuales, sociales y prácticas para vincularse a un entorno universitario, por ejemplo: manejar el dinero en distintos contextos, utilizar el transporte público, llevar a cabo rutinas de higiene y aseo personal, interactuar socialmente con otras personas, desplazarse por diferentes lugares de la ciudad y de la universidad, y reconocer su entorno inmediato.

El programa se fundamentó en los referentes conceptuales de la teoría del socioconstructivismo, centrada en muchas de las ideas de Vygotsky y en la que se plantea que el conocimiento no puede entregarse al estudiante, sino que este es quien elabora sus propias conceptualizaciones y significaciones, las cuales están ligadas al contexto en el cual se aprende, a su historia cultural y a las interacciones sociales que se generan en el proceso de aprendizaje. Desde esta perspectiva, tanto el estudiante como el adulto -mediador- son agentes activos en el proceso de aprendizaje (Verenikina, 2010).

El trabajo didáctico se estructuró utilizando la secuencia didáctica y el taller. La secuencia didáctica "es una estructura de acciones e interacciones relacionadas entre sí, intencionales, que se organizan para alcanzar algún aprendizaje” (Pérez y Rincón, 2009 , p. 7). Se orienta por unos propósitos específicos de enseñanza y aprendizaje planeados por el docente, y vincula saberes conceptuales y prácticos en el contexto de una situación de aprendizaje que le otorga sentido. El taller se caracteriza por que se refiere a un aprender haciendo, en tanto este "se abre como un espacio de experimentación, de puesta en práctica y de producción de soluciones basadas en aprendizajes puntuales o hipótesis fundamentadas en aprendizajes generales" (Pérez et al., 2013, p. 16).

La participación de las familias fue clave en el desarrollo del programa de formación, estas intervenciones se centraron en 1) explorar y conocer un poco el contexto familiar en el que se encontraba inmerso el participante; 2) identificar y reconocer algunas de las prácticas familiares que favorecían u obstaculizaban el fortalecimiento de las habilidades adaptativas en los jóvenes; y 3) generar estrategias de apoyo para las familias que lo requerían, con el propósito de que le permitieran al joven acceder a nuevos aprendizajes y fortalecer sus habilidades adaptativas.

Se propusieron tres modalidades de atención para desarrollar el trabajo con las familias. Los talleres grupales que consistieron en el despliegue de una serie de encuentros periódicos para reflexionar y conversar en torno a algunos contenidos de los módulos formativos; estos tuvieron una frecuencia mensual y en ocasiones bimensual, y se planearon teniendo en cuenta las necesidades e intereses del grupo. Las atenciones focales se realizaron para atender a una necesidad específica del joven y/o de su familia; estas se caracterizaron por ser flexibles y se encontraban en función de algunas necesidades identificadas por el equipo interdisciplinario del programa o por la solicitud directa de la familia. Las derivaciones en servicios especializados consistían en realizar las orientaciones y/o gestiones correspondientes para atender a una necesidad específica de la familia o del joven.

El plan de estudios se estructuró con base en las habilidades de la conducta adaptativa, en tanto estas se consideran necesarias para que una persona en situación de DI sea valorada socialmente y alcance niveles apropiados de autodeterminación. El 
PROPUESTA DIDÁCTICA CON ENFOQUE ECOLÓGICO E INTERDISCIPLINAR:

UNA APUESTA POR LA EDUCACIÓN SUPERIOR INCLUSIVA

D. A. RAMíreZ, P. A. SOTO, M. ${ }^{a}$ T. RUGElES, L. H. LUGO, C. A. QUINTERO, M. ${ }^{a}$ C. OCAMPO ET AL.

modelo de atención integral estuvo compuesto por tres fases de formación: Escalar, UIncluye y Diplomas.

Este modelo de atención integral para las personas en situación de DI se estructuró teniendo en cuenta sus condiciones particulares de salud, sus capacidades intelectuales, y se reconocieron los factores asociados a nivel individual, familiar, cultural y social de cada uno de los jóvenes que participaron en este proceso. De los 31 jóvenes evaluados, se seleccionaron 20 , y a cada uno de ellos se le realizó una evaluación integral para identificar sus condiciones de salud, independencia, y sus habilidades académicas y sociales. De manera particular, se llevó a cabo una entrevista familiar; una evaluación pedagógica para identificar el estado de las habilidades de lectura, escritura y pensamiento lógico-matemático; una evaluación ocupacional y de las condiciones de salud. Los participantes seleccionados demostraron habilidades básicas en los procesos cognitivos de atención, memoria, comprensión, razonamiento, resolución de problemas, y demostraron habilidades sociales para comunicarse, desenvolverse en su cotidianidad e interactuar con su entorno.

La fase Escalar se estructuró con el propósito de preparar a las familias y a los jóvenes para el ingreso a la vida adulta y favorecer su vinculación al entorno universitario. Se desarrolló un plan de trabajo centrado en ocho dimensiones: representaciones sociales; salud y bienestar; ciudadanía y participación; alfabetización; comunicación e interacción; movilidad y contexto; ocio y tiempo libre; y gestión financiera.

En las dos últimas fases: UIncluye y Diplomas, se consolidó e implementó un currículo que incluyó acciones y cursos organizados en tres núcleos: social-práctico, conceptual y vocacional-ocupacional. Cada uno de estos núcleos buscaba la consecución de un objetivo, y para ello se estructuraron una serie de módulos con sus respectivos propósitos de formación, temáticas a abordar, e indicadores de logro que se pretendían fortalecer en los jóvenes en el marco de este programa educativo.

En la fase UIncluye los jóvenes participaron en actividades en estos tres núcleos que se conjugaron con acciones transversales de talleres artísticos y acciones específicas en los siguientes ejes temáticos: Ciencias Alimentarias, Ciencias Veterinarias, Agricultura Urbana y Pedagogía. Este itinerario formativo les permitió a los jóvenes fortalecer su conducta adaptativa; interactuar en espacios de ciudad, y reconocerse como seres humanos que transitan de la adolescencia a la vida adulta, etapa que les demandó nuevos retos y les exigió revisar y ajustar sus roles personales, familiares y sociales. Esta etapa los preparó para asumir con responsabilidad y mayor autonomía su vida adulta consigo mismos, en la familia, en la universidad y en la sociedad en general.

En el núcleo social-práctico se buscó potenciar y favorecer el desarrollo de habilidades sociales, prácticas y de autodirección en los jóvenes, que les permitieran desempeñarse de manera adecuada en una comunidad y que facilitaran su inclusión en el entorno universitario. Este núcleo estuvo integrado por cinco módulos: me identifico, somos un equipo, vida sana, segura e independiente, me relaciono y vivamos la universidad.

En el núcleo conceptual se buscó promover habilidades conceptuales y comunicativas en los jóvenes, que les permitieran minimizar barreras para desempeñarse

Ediciones Universidad de Salamanca / CC BY-NC-ND

Siglo Cero, vol. 52 (3), 2021, julio-septiembre, pp. 163-184 
satisfactoriamente en el entorno familiar, social y universitario. Las habilidades conceptuales incluyen el lenguaje receptivo y expresivo; los procesos de lectura, escritura y pensamiento lógico matemático, al igual que la autodirección, que en este caso se integró en el núcleo social-práctico. El núcleo conceptual estuvo integrado por dos módulos: Alfabetización en la universidad y Creaciones a través del cuerpo y la palabra.

En el núcleo vocacional-ocupacional se estructuraron una serie de talleres con el objetivo de que los participantes comprendieran los conceptos básicos propios de las áreas de formación; aplicaran las acciones y/o funciones propias de estas; incrementaran su autonomía personal; bienestar; fortalecieran sus habilidades para resolver problemas; potenciaran sus habilidades de autodeterminación; y aprendieran a autovalorarse y a mostrarse seguros de sí mismos en el entorno universitario, familiar y social.

Los talleres se llevaron a cabo con la participación de las facultades de artes; medicina veterinaria y ciencias agrarias; y química farmacéutica y ciencias alimentarias. Estos se estructuraron con el objetivo de promover el primer acercamiento de los jóvenes a procesos y contenidos propios del área de énfasis. Con esta experiencia, fue posible evidenciar capacidades, identificar intereses o afinidades de participación en las diferentes áreas abordadas. La característica principal de estos talleres es que fueron exploratorios y le ofrecieron la oportunidad a cada uno de los jóvenes de acercarse a los énfasis de formación.

La sensibilización hacia esta propuesta de formación en las diferentes instancias universitarias se inició en el primer semestre del año 2016. Inicialmente, se hizo el contacto con diferentes unidades académicas y administrativas; en una reunión con las directivas se les presentó el proyecto y la necesidad de tener su apoyo para promover esta iniciativa.

Las áreas de énfasis se seleccionaron teniendo en cuenta la apertura de las unidades académicas de la Universidad de Antioquia. Se vincularon los profesionales que conocen la propuesta y que han decidido apostarle a este proyecto $\mathrm{y}$, a su vez, aportar a su consolidación y dejar de lado los prejuicios existentes, en torno al escepticismo que se produce ante la posibilidad de pensar que estas personas pueden ser protagonistas en la construcción de su propio proyecto de vida (Rocha et al., 2011). Los expertos en cada área se caracterizaron por su disposición para adaptarse a planeaciones más flexibles, en las que se propiciaron y se generaron diferentes espacios para que los jóvenes pudieran explorar y participar de acuerdo con sus posibilidades. Sin embargo, a pesar del alto grado de compromiso que se evidenció por parte de los profesores de los énfasis de formación, se identificó que la falta de conocimientos didácticos y pedagógicos en este campo les dificultó avanzar un poco más en la formación de los jóvenes. Los talleres vocacionales ocupacionales se centraron en el concepto PCP, en tanto se buscó crear los apoyos necesarios teniendo en cuenta los deseos, intereses y particularidades de los jóvenes (Vilá et al., 2012).

Durante la tercera y última fase, denominada Diplomas, se privilegió el desarrollo de dos diplomados, uno en Ciencias Alimentarias y el otro en Ciencias Animales y Agricultura Urbana. El propósito consistió en desarrollar en un grupo de 11 jóvenes algunas capacidades básicas para los procesos de identificación, manipulación y procesamiento de alimentos. Y en los otros 9 jóvenes se fortalecieron algunas capacidades 
PROPUESTA DIDÁCTICA CON ENFOQUE ECOLÓGICO E INTERDISCIPLINAR:

UNA APUESTA POR LA EDUCACIÓN SUPERIOR INCLUSIVA

D. A. RAMÍrEZ, P. A. SOTO, M. $^{a}$ T. RUGElES, L. H. LUGO, C. A. QUINTERO, M. ${ }^{a}$ C. OCAMPO ET AL.

básicas en los procesos de producción, cuidado y atención animal, y en técnicas y adaptación de espacios para la agricultura urbana. Todos los jóvenes participaron en los diplomados durante 90 horas.

\section{Resultados y discusión}

Durante este proceso formativo se identificaron los puntos fuertes y débiles de los jóvenes, y se describieron los apoyos que necesitaban. Esto permitió analizar separadamente todas las áreas en las que los jóvenes presentaban necesidades y, por tanto, requerían de una intervención (Verdugo, 1995). El seguimiento se realizó de manera individual y se tuvieron en cuenta las particularidades de cada joven durante su proceso de formación.

En el desarrollo del proyecto, se identificó que el $100 \%$ de los jóvenes mostraron avances significativos en la construcción de textos más articulados y organizados. El $75 \%$ fortalecieron sus procesos de lectura comprensiva, mientras que el $25 \%$ requirieron que se les apoyara para extraer las ideas centrales de los textos, se les dificultó comprender de manera inferencial un texto y escribir y expresar ideas. En lo que respecta a la comunicación, el $90 \%$ se mostraron más seguros y confiados en el momento de establecer diálogos con otras personas y al $10 \%$ se les dificultó entablar una conversación de manera fluida.

En el área del pensamiento lógico matemático, el 75 \% reconocieron la importancia que tienen los algoritmos de suma, resta, multiplicación y división en la vida cotidiana; además, reconocieron el dinero, componían y descomponían diferentes valores, y realizaban aproximaciones y cálculos para comprar algunos productos; pero al $25 \%$ se les dificultó resolver las operaciones matemáticas, medir el peso de diferentes productos, diferenciar el valor posicional en las cantidades numéricas, y requirieron de apoyos extensos para manejar el dinero, conformar cantidades numéricas y realizar un plan de gastos.

Se identificó que es necesario continuar fortaleciendo las habilidades de autodirección, en tanto se observó que se muestran inseguros en el momento de dar su opinión o comunicar sus decisiones en un grupo de amigos, compañeros o a sus familiares.

A nivel social, el $85 \%$ son capaces de trabajar en grupo y el $15 \%$ presentan dificultades para tener en cuenta la opinión de sus compañeros en el momento de tomar decisiones que los afectan a todos. El $15 \%$ tuvieron dificultades para responsabilizarse de sus compromisos académicos y para comunicarse de manera asertiva con sus compañeros.

En cuanto a las habilidades prácticas, en lo que respecta a los recursos comunitarios, se observó que el 100 \% de los jóvenes lograron desplazarse de forma independiente y fueron capaces de llegar a determinados espacios del campus universitario e hicieron uso de tiendas y cafeterías al interior de la Universidad. Sin embargo, el $50 \%$ requirieron de apoyos extensos para utilizar los servicios de la biblioteca. Se identificaron avances significativos con relación a las habilidades del cuidado de la salud; ya que el $100 \%$ fueron capaces de expresar hábitos de alimentación saludable, 
PROPUESTA DIDÁCTICA CON ENFOQUE ECOLÓGICO E INTERDISCIPLINAR:

UNA APUESTA POR LA EDUCACIÓN SUPERIOR INCLUSIVA

D. A. RAMÍrEZ, P. A. SOTO, M. $^{a}$ T. RUGElES, L. H. LUGO, C. A. QUINTERO, M. ${ }^{a}$ C. OCAMPO ET AL.

refirieron la importancia de la actividad física, expresaron acciones para prevenir y atender algunas enfermedades comunes, y el $45 \%$ lograron identificar y enunciar los riesgos físicos que se pueden presentar en los entornos en los que cotidianamente se desenvuelven.

En cuanto al trabajo vocacional-ocupacional, todos los estudiantes tuvieron la posibilidad de formarse en una de las áreas de énfasis. El $55 \%$ se inclinaron por las ciencias alimentarias y el $45 \%$ profundizaron en ciencias animales y agricultura urbana. En el énfasis de ciencias alimentarias, todos los jóvenes, aunque el $15 \%$ con apoyos intermitentes y extensos, lograron comprender los conceptos abordados y los utilizaron de forma correcta durante la transformación y cocción de alimentos; reconocieron las características de los alimentos mediante su análisis sensorial; y utilizaron herramientas y equipos teniendo en cuenta acciones de prevención de riesgos y la funcionalidad de cada uno. En el énfasis de ciencias animales y agricultura urbana, todos los jóvenes, aunque el $15 \%$ con apoyos intermitentes y extensos, comprendieron los conceptos relacionados con el cuidado y la producción de animales domésticos; identificaron sus características físicas; realizaron acciones de cuidados básicos en los animales domésticos; comprendieron conceptos sobre agricultura urbana; reconocieron y realizaron cada uno de los procesos para el mantenimiento de la ecohuerta, desmalezado, oxigenado, riego, y elaboraron recetas y fusionaron nuevos sabores mezclando plantas alimenticias y medicinales.

En el análisis grupal de la CA, fue posible identificar los avances que presentaron los jóvenes después de su participación en el programa de formación, así como algunas de las dificultades que experimentaron para aprender y poner en práctica determinadas actividades de la vida diaria relacionadas con las dimensiones conceptual, social y práctica.

Como puede observarse en la Figura 1, las puntuaciones obtenidas en la primera y segunda aplicación del instrumento muestran puntuaciones ligeramente superiores después del proceso de intervención ( $\mathrm{Pt} 1$ ). Según el autoinforme aumentó 7,5 puntos el nivel de rendimiento $(\mathrm{p}=0,0028)$, pasando de una mediana de 67,5 a 74,5 ; que los situaba en un nivel de rendimiento muy bajo y lograron avanzar a la categoría baja. A su vez, en el heteroinforme incrementó 4,5 puntos el nivel de rendimiento $(\mathrm{p}=$ 0,0134), y se presentó un cambio de una mediana de 66,5 a 72, logrando avanzar de un nivel de rendimiento muy bajo a bajo.

Después de la primera aplicación del instrumento se encontró que los desempeños de los jóvenes en la vida diaria estaban afectados significativamente, lo que les representaba más barreras para su participación en contexto; mientras que en la segunda aplicación se observaron avances en el desempeño de los jóvenes para realizar diferentes actividades de la vida cotidiana de manera independiente. En este sentido, el proceso formativo que se realizó con los jóvenes y sus familias fortaleció algunos aspectos de la CAG. Este es un hallazgo valioso, dado que, como señalan Verdugo et al. (2012), es necesario planificar programas y estrategias de actuación que les permitan a las personas en situación de DI adaptarse a las demandas de un ambiente social cambiante para asegurar su inclusión y participación en la comunidad. De ahí la importancia de reconocer a todas las personas desde sus capacidades y potencialidades

Ediciones Universidad de Salamanca / CC BY-NC-ND

Siglo Cero, vol. 52 (3), 2021, julio-septiembre, pp. 163-184 
y brindarles los apoyos que necesitan para optimizar su funcionamiento individual (Vicente et al., 2018).

Figura 1. Puntaje en el índice de CAG antes (Pto) y después (pt1) de la participación de los jóvenes en el programa de formación
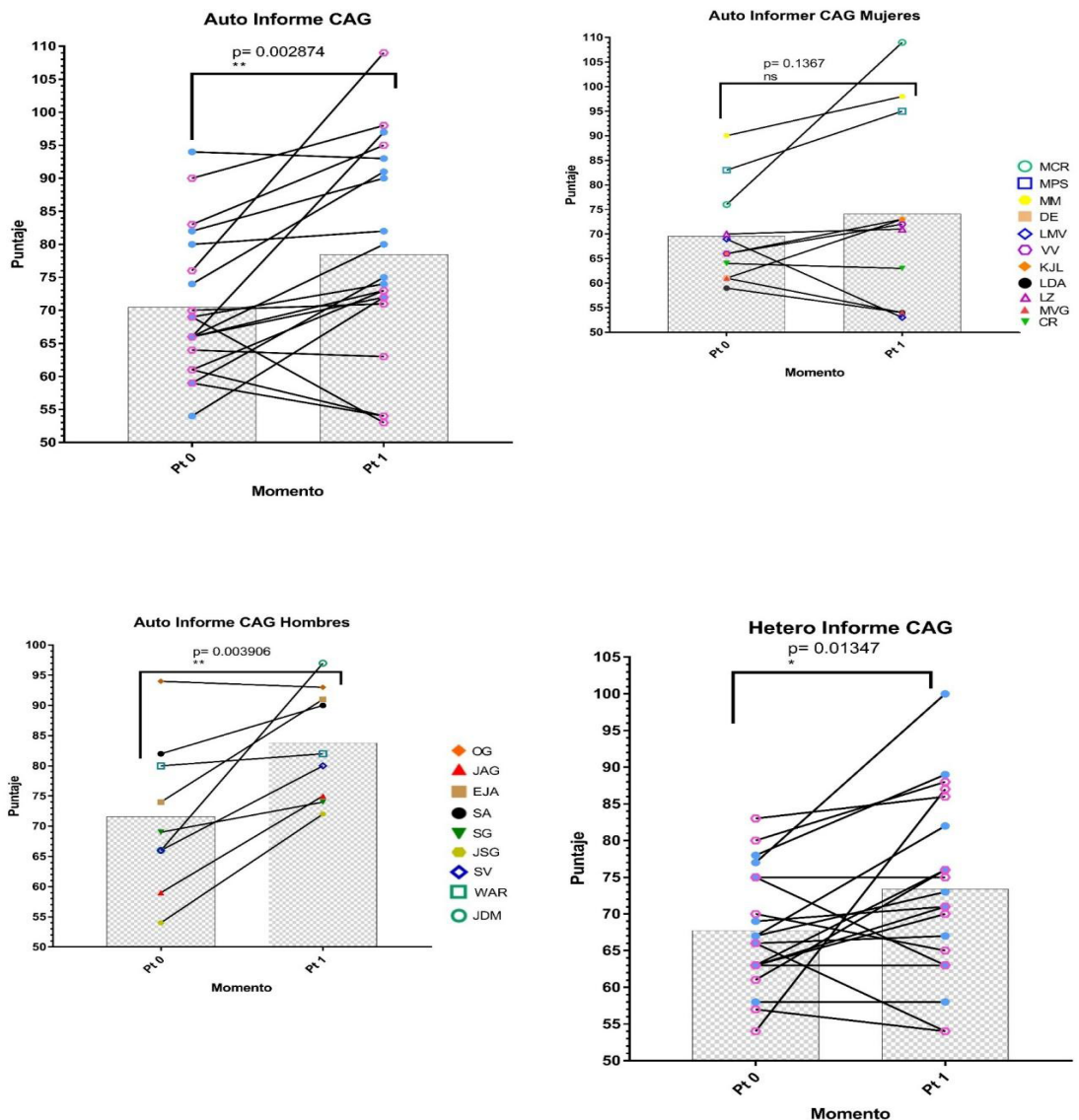

Adicionalmente, se identificó la importancia de poner en marcha estrategias que favorezcan una educación planificada, continua y que se oriente hacia la implementación de apoyos sistemáticos tanto en los niveles de la educación básica y media como en la educación superior. Es necesario realizar transiciones efectivas entre los diferentes niveles de la educación, lo que supone recibir los apoyos para asegurar un tránsito adecuado de las personas en situación de DI, desde el aprendizaje escolar hasta la formación superior y su incorporación al entorno laboral (Alcaín y Medina, 2017). 
PROPUESTA DIDÁCTICA CON ENFOQUE ECOLÓGICO E INTERDISCIPLINAR:

UNA APUESTA POR LA EDUCACIÓN SUPERIOR INCLUSIVA

D. A. RAMíreZ, P. A. SOTO, M. ${ }^{a}$ T. RUGElES, L. H. LUGO, C. A. QUINTERO, M. ${ }^{a}$ C. OCAMPO ET AL.

\section{Conclusiones}

Es posible estructurar propuestas de formación orientadas a favorecer el desarrollo social, académico, familiar y ocupacional de los jóvenes en situación de DI, teniendo en cuenta sus potencialidades y no sus limitaciones, en tanto se ha demostrado que sus posibilidades de aprendizaje superan las que se les han reconocido históricamente (Ramírez, 2002). Además, es necesario que los maestros de todos los niveles educativos estén informados, sensibilizados y formados para atender a las necesidades de todos los estudiantes (Chaves, 2018; Moriña y Carballo, 2018).

Con respecto a los apoyos que requirieron los jóvenes para desarrollar las actividades, se observó que cada uno presentó tanto habilidades como dificultades distintas en intensidad en relación con sus demás compañeros. En este contexto, se reconoce que la educación representa el principal recurso para que todas las personas tengan una vida más autónoma y fortalezcan sus competencias (Muntaner, 2010). La educación puede habilitar o ignorar algunas formas de ser de los sujetos, por ello, es necesario reconocer que a todas las personas se les puede enseñar, pero que cada una aprende en su singularidad (Skliar, 2015).

A su vez, se identificó que es necesario continuar fortaleciendo el papel de las familias, para que acompañen a los jóvenes sin caer en la sobreprotección o en la limitación de sus habilidades para participar, tomar decisiones y resolver problemas. Durante este proceso fue necesario formar a las familias, ya que algunas tomaban las decisiones por sus hijos, se les dificultaba verlos como seres independientes y no les permitían ejercer sus derechos (Cuesta et al., 2019).

El ABAS-II es un instrumento que puede ser utilizado con la población en situación de DI de forma complementaria para determinar los logros y dificultades en la CA. La escala proporciona una base muy sólida sobre la cual planificar intervenciones basadas en la persona en diversos contextos, y su evaluación puede ayudar a mejorar su calidad de vida en cualquier momento de su ciclo vital (Harrison y Oakland, 2013).

Finalmente, las universidades están llamadas a respetar la diferencia y a acoger los marcos legales que regulan la atención educativa que se le ofrece a la población en situación de discapacidad, para que de esta manera se pueda avanzar en el proceso de equiparación de oportunidades de los jóvenes que desean vincularse a los estudios superiores.

\section{Referencias bibliográficas}

Agran, M., Spooner, F. y ZaKas, T. (2013). La salud y la seguridad como áreas de habilidad adaptativa. En P. HARRISON y T. OAKLAND (Eds.), AbAs-II. Uso clinico e interpretación (pp. 201-230). Tea Ediciones.

Alcaín, E. y Medina, M. (2017). Hacia una educación universitaria inclusiva: realidad y retos. Revista Digital de Investigación en Docencia Universitaria, 11(1), 4-19. https://doi. org/10.19083/ridu.11.530 
PROPUESTA DIDÁCTICA CON ENFOQUE ECOLÓGICO E INTERDISCIPLINAR:

UNA APUESTA POR LA EDUCACIÓN SUPERIOR INCLUSIVA

D. A. RAMÍrEZ, P. A. SOTO, M. $^{a}$ T. RUGElES, L. H. LUGO, C. A. QUINTERO, M. ${ }^{a}$ C. OCAMPO ET AL.

Arellano, A. y Peralta, F. (2016). La planificación centrada en la persona: un ejemplo de buena práctica en el ámbito de la discapacidad intelectual. Contextos Educativos, 19, 195292. https://doi.org/10.18172/con.2754

Barradas, M. E., Valerio, C., Pérez, J., Gutiérrez, V. y Saldivar, G. (2019). Políticas públicas y desigualdad en educación superior. Revista Electrónica sobre Educación Media y Superior, 6(11), 1-26. https://bit.ly/38yvw9F

Bernal, A. (2014). Universidad e inclusión de personas con discapacidad. Paidea Surcolombiana, 2(19), 55-61. https://doi.org/10.25054/01240307.1166

Carratalá, A., Mata, G. y Crespo, S. (2017). Planificación Centrada en la Persona. Planificando por adelantado el Futuro Deseado. Plena inclusión. https://bit.ly/2itTbxi

Chaves, C. F. (2018). La inclusión de personas con diversidad funcional en la educación superior. Hojas y Hablas, 16, 84-95. https://doi.org/10.29151/hojasyhablas.n16a6

Claro, J. P. (2007). Estado y desafíos de la inclusión educativa en las Regiones Andina y Cono Sur. Revista Iberoamericana sobre Calidad, Eficacia y Cambio en Educación, 5(5), 179-187. https://bit.ly/2UeE6f7

Cobos, A. y Moreno, M. (2014). Educación superior y discapacidad: análisis desde la experiencia de algunas universidades colombianas. Revista Española de Discapacidad, 2(2), 83-101. https://dx.doi.org/10.5569/2340-5104.02.02.05

Congreso De la República De Colombia. (31 de julio de 2009). Ley 1346 por medio de la cual se aprueba la "Convención sobre los Derechos de las personas con Discapacidad". https://bit.ly/32Asnck

CotÁn FernándeZ, A. (2017). Educación inclusiva en las instituciones de educación superior: narrativas de estudiantes con discapacidad. Revista Española de Discapacidad, 5(1), 43-61. https://doi. org/10.5569/2340-5104.05.01.03

CRESWELL, W. J. (2013). Research design: qualitative, quantitative, and mixed methods approaches. SAGE.

Cuervo, T., Iglesias, M. T. y Fernández, S. (2017). Efectos y beneficios de la planificación centrada en la persona (РСP) en las personas adultas con discapacidad intelectual, en las organizaciones y en la comunidad. Estado de la cuestión. Siglo Cero, 48(2), 25-40. https:// doi.org/10.14201/scero20174822540

Cuesta, J. L., De la Fuente, R. y Ortega, T. (2019). Discapacidad intelectual: una interpretación en el marco del modelo social de la discapacidad. Controversias y Concurrencias Latinoamericanas, 10(18), 85-106. https://bit.ly/2GPykgG

Delgado, G. (2015). Rechazo social a alumnos con discapacidad intelectual en educación primaria. [Tesis de pregrado, Universidad de Valladolid, España]. https://uvadoc.uva.es/ handle/10324/14837

Díez, E. y SÁnchez, S. (2015). Diseño Universal para el Aprendizaje como metodología docente para atender a la diversidad en la universidad. Aula Abierta, 43(2), 87-93. https://doi. org/10.1016/j.aula.2014.12.002

Dymond, S. K. (2013). La conducta y las habilidades adaptativas importantes para la utilización de los recursos comunitarios. En P. Harrison y T. OAKLAND (Eds.), Abas-II. Uso clínico e interpretación (pp. 101-133). Tea Ediciones.

Flórez, J. (2018). La comprensión actual de la discapacidad intelectual. Sal Terrae, 106(1234), 479-492. https://bit.ly/3erJr5e

Harrison, P., y OAKLAND, T. (2013). Introducción a las conductas y habilidades adaptativas. En Abas-II. Uso clínico e interpretación (pp. 13-37). Tea Ediciones.

Ediciones Universidad de Salamanca / CC BY-NC-ND

Siglo Cero, vol. 52 (3), 2021, julio-septiembre, pp. 163-184 
PROPUESTA DIDÁCTICA CON ENFOQUE ECOLÓGICO E INTERDISCIPLINAR:

UNA APUESTA POR LA EDUCACIÓN SUPERIOR INCLUSIVA

D. A. RAMÍrEZ, P. A. SOTO, M. $^{a}$ T. RUGElES, L. H. LUGO, C. A. QUINTERO, M. ${ }^{a}$ C. OCAMPO ET AL.

Hernández, S., Fernández, C. y Baptista, L. (2010). Metodología de la investigación. McGraw Hill.

Jiménez, A. y Verdugo, M. Á. (1999). Situación de las personas con discapacidad en Castilla La Mancha: las necesidades percibidas. En F. B. Jordán De Urríes y M. Á. Verdugo (Coords.), Hacia una nueva concepción de la discapacidad. Actas de las III Jornadas Científicas de Investigación sobre Personas con Discapacidad (pp. 299-308). Amarú Ediciones.

Jordán De Urríes, F. B. y Verdugo, M. Á. (2013). Empleo integrado, la llave para la vida adulta. En M. Á. Verdugo y R. L. SCHALOck (Eds.), Discapacidad e inclusión. Manual para la docencia (pp. 379-403). Amarú Ediciones. https://bit.ly/2uk6wuc

Kremer, S. F. y Quijano, O. (2015). Prácticas pedagógicas, promoción de inclusión y diversidad en una institución educativa de Popayán. Plumilla Educativa, 15(1), 67-86. https://doi. org/10.30554/plumillaedu.15.833.2015

Miller, M. y Fenty, N. (2013). Habilidades académicas funcionales. En P. Harrison y T. OAKLAND (Eds.), Abas-Ir. Uso clínico e interpretación (pp. 135-165). Tea Ediciones.

Ministerio De Educación Nacional De Colombia. (1998). Serie lineamientos curriculares. Indicadores de logros curriculares. https://bit.ly/2104Hnc

Ministerio De Educación Nacional De Colombia. (2006). Estándares básicos de competencias en matemáticas. Potenciar el pensamiento matemático. ;Un reto escolar! https://bit. ly/38An7fi

Ministerio De Educación Nacional De Colombia. (2007). Articulación de la educación con el mundo productivo. Competencias laborales generales. Men. https://bit.ly/3kuUeux

Ministerio De Educación Nacional De Colombia. (2017). Decreto 1421. Por el cual se reglamenta en el marco de la educación inclusiva la atención educativa de la población con discapacidad. https://bit.ly/32RAz1t

Moriña, A. y Carballo, R. (2018). Professorado universitário e educação inclusiva: respondendo a suas necessidades de formação. Psicología Escolar e Educacional, 22, 87-95. https:// doi.org/10.1590/2175-35392018053

MoriñA, A. y CotÁn, A. (2017). Educación Inclusiva y Enseñanza Superior desde la mirada de estudiantes con diversidad funcional. Revista Digital de Investigación en Docencia Universitaria, 11(1), 17-34. https://doi.org/10.19083/ridu.11.528

Muntaner, J. (2010). Escuela y discapacidad intelectual. Propuestas para trabajar en el aula ordinaria. Ediciones de la U.

Mutua, K. (2013). Habilidades y actitudes relacionadas con el empleo. En P. Harrison y T. OAKLAND (Eds.), Abas-Ii. Uso clínico e interpretación (pp. 315-348). Tea Ediciones.

Ocampo, A. (2013). Inclusión de estudiantes en situación de discapacidad a la educación superior. Desafíos y oportunidades. Revista Latinoamericana de Educación Inclusiva, 6(2), 227-239. https://bit.ly/36vc2Ju

Pallisera, M. (2011). La inclusión laboral y social de los jóvenes con discapacidad intelectual. El papel de la escuela. Revista Interuniversitaria de Formación del Profesorado, 70, 185200. https://bit.ly/3nfhc3I

Pallisera, M., Fullana, J., Vilá, M., Jiménez, P., Castro, M., Puyalto, C., Moreno, M., y Martín, R. (2014). Análisis de los apoyos que reciben los jóvenes con discapacidad intelectual en su transición a la vida adulta en España: una investigación a partir de experiencias de profesionales y personas con discapacidad. Revista Española de Discapacidad, 2(2), 27-43. https://dx.doi.org/10.5569/2340-5104.02.02.02

Park, K. L., Loman, S. y Miller, M. A. (2013). Habilidades sociales. En P. Harrison y T. OAKLAND (Eds.), Abas-Ii. Uso clínico e interpretación (pp. 285-313). Tea Ediciones.

Ediciones Universidad de Salamanca / CC BY-NC-ND

Siglo Cero, vol. 52 (3), 2021, julio-septiembre, pp. 163-184 
PROPUESTA DIDÁCTICA CON ENFOQUE ECOLÓGICO E INTERDISCIPLINAR:

UNA APUESTA POR LA EDUCACIÓN SUPERIOR INCLUSIVA

D. A. RAMÍrEZ, P. A. SOTO, M. $^{a}$ T. RUGElES, L. H. LUGO, C. A. QUINTERO, M. ${ }^{a}$ C. OCAMPO ET AL.

Pérez, M. y Rincón, G. (2009). Actividad, secuencia didáctica y pedagogía por proyectos: tres alternativas para la organización del trabajo didáctico en el campo del lenguaje. CERLALC.

Pérez, M., RoA, C., Villegas, L. y VARgas, A. (2013). Escribir las prácticas: una propuesta metodológica para planear, analizar, sistematizar y publicar el trabajo didáctico que se realiza en las aulas. Pontificia Universidad Javeriana.

Ramírez, D. A. (2002). Comprensión lectora en adolescentes con síndrome de Down. Revista Educación y Pedagogía, 14(33), 145-158. https://bit.ly/3enMTxU

Ramírez, M. (2018). Las prácticas realizadas por la Universidad de Costa Rica, para garantizar la educación superior de las personas con discapacidad. Revista Reflexiones, 97(2), 35-54. https://dx.doi.org/10.15517/rr.v97i2.32948.

Rocha, M., Capel, G., Durand, F., Gómez, A. y Balero, D. (2011). Estado de situación de las prácticas de Orientación Vocacional Ocupacional en sujetos con discapacidad en la República Argentina. En XI Jornadas Argentinas de Estudios de Población Ciudad de Neuquén. https://bit.ly/3nhoyw6

Simons, H. (2011). El estudio de caso: teoría y práctica. Morata.

SKLIAR, C. (2015). Preguntar la diferencia: cuestiones sobre la inclusión. Revista Sophia, 11(1), 33-43. https://bit.ly/3pmc9an

Steere, D., Garrison, H. y Burgender, J. (2013). Habilidades adaptativas de la vida en el hogar y en la escuela. En P. Harrison y T. OAKLAND (Eds.), Abas-Ii. Uso clínico e interpretación (pp. 167-200). Tea Ediciones.

TAmarit, J. (2010). La calidad como presupuesto de los servicios de apoyo a las personas con discapacidad. En L. Cayo (Dir.), Discapacidad, Tercer Sector e Inclusión social (pp. 311338). Ediciones Cinca.

Verdugo, M. Á. (1995). Las personas con retraso mental. En A. Aguado y M. Á. Verdugo (Coords.), Personas con discapacidad. Perspectivas psicopedagógicas y rehabilitadoras (pp. 515-554). Siglo Veintiuno.

Verdugo, M. Á., Navas, P., Jordán De Urríes, F. B., Gómez, L. E. y Arias, B. (2012). Evaluación de la conducta adaptativa en el contexto español. En S. SANTOS y P. Morato (Eds.), Comportamento adaptativo. Dez anos depois (pp. 35-52). Faculdade de Motricidade Humana. https://bit.ly/32y63cu

Verenikina, I. (2010). Vygotsky in twenty-first-century research. En J. Herrington y B. Hunter (Eds.), Proceedings of World Conference on Educational Multimedia, Hypermedia and Telecommunications (pp. 16-25). AACE.

Vicente-Sánchez, E., Guillén-Martín, V. M., Verdugo-Alonso, M. Á. y Calvo-ÁlvaREZ, M. I. (2018). El rol de los factores personales y familiares en la autodeterminación de jóvenes con discapacidad intelectual. Psicología Educativa, 24(2), 75-83. https://doi. org/10.5093/psed2018a13

Vicente-Sánchez, E., Mumbardó-Adam, C., Coma, T., Verdugo, M. Á. y Giné, C. (2018). Autodeterminación en personas con discapacidad intelectual y del desarrollo: revisión del concepto, su importancia y retos emergentes. Revista Española de Discapacidad, 6(2), 7-25. https://doi.org/10.5569/2340-5104.06.02.01

Vilá, M., Pallisera, M. y Fullana, J. (2012). La inclusión laboral de los jóvenes con discapacidad intelectual: un reto para la orientación psicopedagógica. Revista Española de Orientación y Psicopedagogía, 23(1), 85-93. https://bit.ly/32Av00I

Vived, E., Betbesé, E. y Díaz, M. (2012). Formación para la inclusión social y la vida independiente. Sindrome de Down: Vida Adulta, 4(11), 34-42. https://bit.ly/3kmmqJ1 
PROPUESTA DIDÁCTICA CON ENFOQUE ECOLÓGICO E INTERDISCIPLINAR: UNA APUESTA POR LA EDUCACIÓN SUPERIOR INCLUSIVA

D. A. RAMírez, P. A. SOTO, M. ${ }^{a}$ T. RUGElES, L. H. LUGO, C. A. QUiNTERO, M. ${ }^{a}$ C. OCAMPO ET AL.

Wallace, M. y Shubert, M. (2013). La estimulación de las habilidades de autocuidado. En P. Harrison y T. OAKLand (Eds.), Abas-II. Uso clínico e interpretación (pp. 261-284). Tea Ediciones.

Zacarías, J., SAad, E., Fiorentini, Z. y Nava, E. (2015). Los significados de la inclusión a la universidad en jóvenes con discapacidad intelectual: análisis de casos. En IX Jornadas Científicas Internacionales de Investigación sobre Personas con Discapacidad (pp. 1-11). https://bit.ly/3ixz9o1

Ediciones Universidad de Salamanca / CC BY-NC-ND

Siglo Cero, vol. 52 (3), 2021, julio-septiembre, pp. 163-184 